\title{
基于统计过程控制的流程工业工艺规范制定方法*
}

\author{
徐 钢 ${ }^{1,2}$ 张晓殁 ${ }^{1}$ 黎 敏 ${ }^{2}$ 徐金梧 $^{2}$ \\ (1. 北京科技大学计算机通讯工程学院 北京 100083 ; \\ 2. 北京科技大学钢铁共性技术协同创新中心 北京 100083)
}

\begin{abstract}
摘要: 在冶金、化工等流程型工业领域, 产品制造过程涉及多个连续相关的工序, 每道工序需确定工艺参数的控制范围, 即 制定工艺规范。目前, 流程工业在制定工艺规范时, 主要采用 6-Sigma 方法或根据生产经验来确定每道工序关键工艺参数的 范围。但是, 这种基于独立同分布假设的 6-Sigma 方法, 由于缺乏处理多重相关、非正态分布的能力, 因此所制定的工艺规 范在工业应用中容易造成产品质量的不稳定。为了解决流程工业在工艺规范制定过程中存在的这个难题, 提出了基于多元统 计分析原理的工艺规范制定方法。首先, 引入了软超球体的概念, 并采用非线性核函数的方法来确定软超球体在高维空间中 的边界, 即产品质量可控区的工艺参数边界。然后, 寻求软超球体的最大内接矩形体, 并依据最大内接矩形体来制定工艺规 范。最后, 结合 IF 钢生产实例, 讨论了工艺规范制定的整个过程, 验证了新方法的有效性。
\end{abstract}

关键词: 流程工业; 工艺规范; 软超球体; 多元统计过程控制

中图分类号: TP277

\section{A Method of Establishing Process Specifications in Process Industry Based on Statistical Process Control}

\author{
XU Gang $^{1,2}$ ZHANG Xiaotong ${ }^{1} \quad$ LI Min $^{2} \quad$ XU Jinwu $^{2}$ \\ (1. School of Computer and Communication Engineering, \\ University of Science and Technology Beijing, Beijing 100083; \\ 2. Collaborative Innovation Center of Steel Technology, \\ University of Science and Technology Beijing, Beijing 100083)
}

\begin{abstract}
In the field of process industry such as metallurgy and chemical industry, the manufacturing process of the product involves several continuous related processes. Each process needs to determine the control range of the process parameters, that is, the process specification is formulated. At present, the process industry mainly uses the 6-Sigma method or the production experience to determine the range of key process parameters in each process. However, the 6-Sigma method based on the assumption of independent distribution is lacking in the ability to deal with multiple correlation and non-normal distribution, so the process specification is easy to cause the instability of product quality in industrial application. In order to solve the problem existing in the process specification of the process industry, a method for formulating process specifications based on multivariate statistical analysis is proposed. First, the concept of soft hyper-sphere is introduced, and the method of nonlinear kernel function is used to determine the boundary of the soft hyper-sphere in the high dimensional space, that is the process parameter boundary of the product quality-controlled zone. Then, the largest inscribed form of the hyper-sphere is found out, and the process specification is formulated according to the largest inscribed rectangle. Finally, the example of IF steel production is conducted, and the formulation process of the process specification is discussed, so the effectiveness of the new method is verified.
\end{abstract}

Key words: process industries; process specifications; soft hyper-sphere; multivariate statistical process control

\section{0 前言}

在钢铁、石化等流程工业领域, 在制造过程中
需要确定各工序的工艺参数控制范围, 即针对不同 系列产品制定各工序的工艺规范。如果工艺参数(包 括原料参数)控制在工艺规范范围内, 认为所设定的 过程参数能满足产品质量要求, 反之, 则可能出现 质量异常。目前, 流程工业在制定工艺规范时, 主 要采用 6-Sigma 方法或根据生产经验来确定每道工
“十二五” 国家科技支撑计划资助项目(2015BAF30B01)。20180625 收 到初稿, 20181120 收到修改稿 
序关键工艺参数的范围。这种通过实际生产数据来 制定产品的工艺规范, 并按照规范要求对产品质量 实施有效控制, 在工业生产中发挥了重要的作用 ${ }^{[1]}$ 。

但是, 这种基于参数独立同分布假设的 6-Sigma 方法并不适用于多重相关、非正态分布的数据统计 分析, 所制定的工艺规范容易造成产品质量不稳定。 采用 6-Sigma 方法主要针对每个单一独立变量且正 态分布的统计过程, 但在实际工业生产中, 工艺参 数间存在着不同程度的相关性, 而单一独立变量统 计过程控制忽略了变量间的相关性, 因此难以准确 判别生产过程中的复杂行为。

为了解决变量间多重相关问题, 提出了多变量 统计过程控制(Multivariate statistical process control, MSPC)方法, 实现多变量生产过程的质量监控 ${ }^{[2-5]}$ 。 传统的多变量统计过程的前提是, 对于一个稳定的 生产过程, 正常样本点分布在高维空间中的某个超 椭球体内; 一旦样本点超出超椭球体的边界, 可以 认为该生产过程出现了异常。多变量统计过程控制 的实质是通过历史数据来确定高维空间中这个超椭 球的位置和大小。但是在实际工业生产过程中, 尤 其对于冶金、化工这类复杂流程工业，工艺变量间 往往存在多重相关和非正态分布的数据结构特征, 数据分布在一个软性的超球体内, 即超球体的边界 是一组复杂的超曲面 ${ }^{[6]}$ 。对于这类复杂的数据结构, 采用传统的超椭球边界的方法来判别异常点会造成 大量的误判。针对工业生产中常见的这类复杂的数 据结构, 引入软超球体的概念, 并采用非线性核函 数的方法来确定软超球体在高维空间上的复杂边 界, 即产品质量可控区的工艺参数边界。然后, 寻 求软超球体的最大内接矩形体, 并依据最大内接矩 形体来制定工艺规范。结合某类汽车用钢在制造过 程中的工业应用实例, 讨论了工艺规范制定的整个 过程以及需注意的相关问题。

\section{1 工艺参数可控区范围的确定}

由于工艺变量间存在多重相关和非正态分布的 数据结构, 数据在高维空间中的分布通常是一个软 超球体, 因此凭借参数独立同分布假设的 6-Sigma 方法所确定的工艺规范会造成产品质量的偏差。为 了解决流程工业中常出现的这个问题, 首先引入软 超球体的概念, 并讨论如何从数据集中确定软超球 体的边界。来自于同一类数据的空间边界划分方法 也称为单一类分类问题 ${ }^{[6]}$ 。

\section{1 质量可控区边界}

假设给定一个数据集 $S=\left\{x_{1}, x_{2}, \cdots, x_{n}\right\}$, 其中 $x_{i}$
为 $p$ 维的数据向量, 需确定该数据集的边界, 即求解 包含该数据集的最小封闭软超球体的边界。但是, 由 于在实际工业生产中, 质量异常点与正常点的部分边 界有可能是交叉重叠的, 因此需要考虑允许少数正常 样本点被划在边界外。通常, 包含绝大部分满足质量 要求的工艺参数样本点的封闭超球体称为软超球 体 ${ }^{[7-8]}$, 它是在硬球体的基础上对边界作了松弛处理, 即允许少数质量正常的样本点被划在边界外。图 1 给 出了二维空间中数据点分布对边界划定的影响。

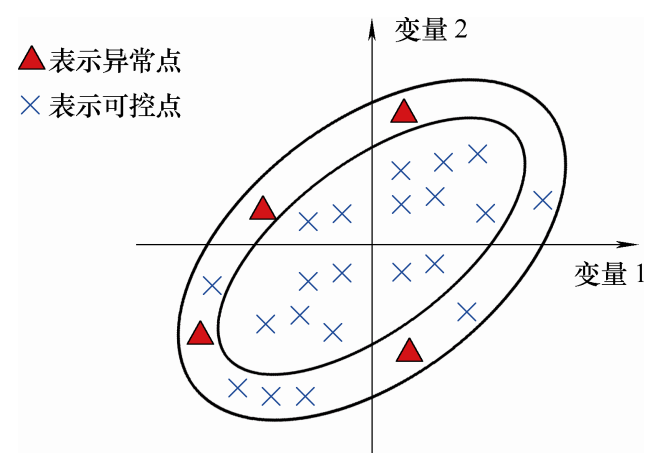

图 1 封闭软超球体

封闭软超球体的优化解为

$$
\min _{r, \xi} r^{2}+A\|\xi\|_{1}
$$

约束条件为

$$
\begin{gathered}
\left\|\boldsymbol{x}_{i}-\boldsymbol{C}\right\|^{2}=\left(\boldsymbol{x}_{i}-\boldsymbol{C}\right)^{\mathrm{T}}\left(\boldsymbol{x}_{i}-\boldsymbol{C}\right) \leqslant r^{2}+\xi_{i} \\
\xi_{i} \geqslant 0, \quad i=1,2, \cdots, n
\end{gathered}
$$

式中, $C$ 表示超球体的中心, $r$ 为超球体的半径, $n$ 为样本个数, $\|\xi\|_{1}=\sum_{i=1}^{n} \xi_{i}$ 为一常数, 其值需在控制 半径最大化与控制松驰变量之间做出权衡。常数 $A$ 的选择与第一类错误和第二类错误有关 ${ }^{[9]}$, 通常 $A$ 可以取 0.1 或 0.05 。

引入拉格朗日乘子 $\alpha_{i} \geqslant 0, \beta_{i} \geqslant 0$, 式(1)的拉格 朗日函数为

$$
\begin{gathered}
L(C, r, \alpha, \xi)=r^{2}+A \sum_{i=1}^{n} \xi_{i}- \\
\sum_{i=1}^{n} \beta_{i} \xi_{i}+\sum_{i=1}^{n} \alpha_{i}\left[\left\|\boldsymbol{x}_{i}-C\right\|^{2}-r^{2}-\xi_{i}\right]
\end{gathered}
$$

分别对式(2)求 $\boldsymbol{C}, r, \xi_{i}$ 的偏导, 且令导数值为 0 , 求得下面的优化解

$$
\begin{aligned}
& \frac{\partial L(\boldsymbol{C}, r, \alpha, \xi)}{\partial \boldsymbol{C}}=2 \sum_{i=1}^{n} \alpha_{i}\left(\boldsymbol{x}_{i}-\boldsymbol{C}\right)=0 \\
& \frac{\partial L(\boldsymbol{C}, r, \alpha, \xi)}{\partial r}=2 r\left(1-\sum_{i=1}^{n} \alpha_{i}\right)=0 \\
& \frac{\partial L(\boldsymbol{C}, r, \alpha, \xi)}{\partial \xi_{i}}=A-\alpha_{i}-\beta_{i}=0
\end{aligned}
$$


从而得到

$$
\sum_{i=1}^{n} \alpha_{i} \boldsymbol{x}_{i}=C, \sum_{i=1}^{n} \alpha_{i}=1, \beta_{i}=A-\alpha_{i} \geqslant 0, \alpha_{i} \leqslant A
$$

将这些约束条件代入式(2)，有

$$
\begin{gathered}
L(\boldsymbol{C}, r, \alpha, \xi)=r^{2}+A \sum_{i=1}^{n} \xi_{i}+\sum_{i=1}^{n} \alpha_{i}\left[\left\|\boldsymbol{x}_{i}-\boldsymbol{C}\right\|^{2}-r^{2}-\xi_{i}\right]- \\
\sum_{i=1}^{n} \beta_{i} \xi_{i}=\sum_{i=1}^{n} \alpha_{i}\left\langle\boldsymbol{x}_{i}-\boldsymbol{C}, \boldsymbol{x}_{i}-\boldsymbol{C}\right\rangle= \\
\sum_{i=1}^{n} \alpha_{i} \kappa\left(\boldsymbol{x}_{i}, \boldsymbol{x}_{i}\right)-\sum_{i, j=1}^{n} \alpha_{i} \alpha_{j} \kappa\left(\boldsymbol{x}_{i}, \boldsymbol{x}_{j}\right)
\end{gathered}
$$

令

$$
\omega(\alpha)=\sum_{i=1}^{n} \alpha_{i} \kappa\left(\boldsymbol{x}_{i}, \boldsymbol{x}_{i}\right)-\sum_{i=1}^{n} \sum_{j=1}^{n} \alpha_{i} \alpha_{j} \kappa\left(\boldsymbol{x}_{i}, \boldsymbol{x}_{j}\right)
$$

则, 式(1)转化为求下面优化解

$$
\begin{aligned}
& \max _{\alpha} \omega(\alpha) \\
& \sum_{i=1}^{n} \alpha_{i}=1, \quad 0 \leqslant \alpha_{i} \leqslant A=1 / \gamma n, \quad \gamma=1 / A n
\end{aligned}
$$

由式(5)可以求得最小超球体的半径 $r$ 和球心 $\boldsymbol{C}$

$$
\begin{aligned}
& r=\sqrt{\kappa\left(x_{i}, x_{i}\right)-2 \sum_{j=1}^{n} \alpha_{i}^{*} \kappa\left(x_{i}, x_{j}\right)+\sum_{i=1}^{n} \sum_{j=1}^{n} \alpha_{i}^{*} \alpha_{j}^{*} \kappa\left(x_{i}, x_{j}\right)} \\
& \boldsymbol{C}=\sum_{i=1}^{n} \alpha_{i}^{*} x_{i}
\end{aligned}
$$

式中, $\alpha_{i}^{*}$ 是由式(5)求得的最优解。样本点 $x$ 到超球 体的球心 $C$ 之间的距离 $d(x)$

$$
d(\boldsymbol{x})=\sqrt{\kappa(\boldsymbol{x}, \boldsymbol{x})-2 \sum_{j=1}^{n} \alpha_{i}^{*} \kappa\left(\boldsymbol{x}, \boldsymbol{x}_{j}\right)+\sum_{i=1}^{n} \sum_{j=1}^{n} \alpha_{i}^{*} \alpha_{j}^{*} \kappa\left(\boldsymbol{x}_{i}, \boldsymbol{x}_{j}\right)}
$$

需要指出的是, 式(5)仅适用于工艺参数满足独 立同分布情况, 但在实际工业生产中, 由于过程参 数间的多重耦合，数据中不可避免的存在非正态、 非线性分布现象。因此, 处理这类高维复杂边界需 要采用非线性核函数方法 ${ }^{[10-15]}$ 。核函数方法将原始 空间的样本点映射到高维特征空间中, 且通过计算 映射到特征空间中的样本点间的内积, 构建核矩阵, 并将式(7)中的线性内积项 $\kappa\left(\boldsymbol{x}_{i}, \boldsymbol{x}_{j}\right)$ 替换为非线性核 函数的内积项。在计算核矩阵的每个元素值时, 实 际上并不需要知道样本点映射到特征空间中的具体 数值大小, 而是将原始空间中的样本点代入到函数 中直接计算即可得到内积值 ${ }^{[10]}$ 。

常用的核函数有线性核函数、多项式核函数、 高斯核函数、Sigmoid 核函数。在实际应用中, 由 于高斯核函数是高次级数展开的极限形式, 它可以
表达线性核、多项式核等其他核函数，尤其适用于 复杂非线性的数据结构。

高斯核函数定义为

$$
k(\boldsymbol{x}, \mathbf{z})=\exp \left(-\frac{\|\boldsymbol{x}-\boldsymbol{z}\|^{2}}{\sigma}\right)
$$

当核参数 $\sigma$ 取较大值时, 高次项会迅速衰减, 退化 为线性核函数; 当核参数 $\sigma$ 取较小值时, 高次项的 影响将更加突出, 但容易造成过拟合。

图 2 给出了 “香蕉型” 仿真数据的软球体在 $X-Y$ 平面上投影图, 其中 $\sigma=3$ 对应于复杂的边界, $\sigma=8$ 对应于椭圆边界。从图中可以看出, 当核参数 $\sigma=3$ 时, 数据边界内包含了绝大部分的正常数据, 较正 确地划分了数据边界; 而当核参数 $\sigma=8$ 时, 边界呈 椭圆, 且将边界在 $X$ 轴上延伸了部分空间, 但拓展 部分将可能造成第二类误判, 即将质量异常区误判 为质量合格区。

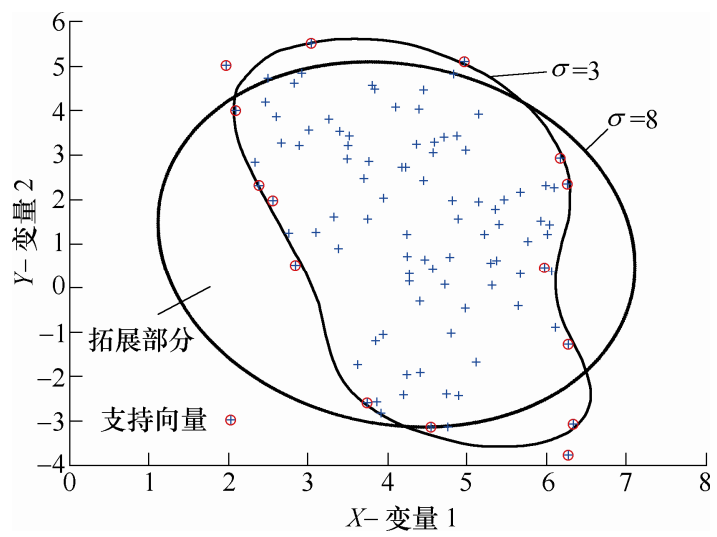

图 2 软球体在 $X-Y$ 平面上投影图

实际上, 前面讨论的超球体内多数样本点的 $\alpha_{i}=0$, 仅在边界附近的点 $\alpha_{i}>0$, 这些点称为支持 向量 ${ }^{[7-8]}$, 如图 2 中圆圈点所示。此外, 需要说明的 是, 从低维空间映射到高维空间的过程中, 并不会 改变样本点之间的邻近关系, 即在原始空间中邻近 的两个样本, 在高维空间中依然是邻近的。因此, 从低维空间到高维空间的映射, 并不改变数据的分 布规律, 而是能够更好地挖掘数据在高维空间中的 本征结构。由此可知, 在高维空间中的软椭球边界 是原始空间中数据边界的高维表征, 两者之间在数 学上存在着一一对应关系。综上所述, 利用高维空 间中的软椭球边界来反映实际生产过程中的质量可 控区是可行的。

\section{2 工艺规范的确定}

实际上，工艺规范的制定过程是从软超球体 中寻求最大的内接矩形体。对于流程工业, 如钢 铁工业, 钢材在生产过程中涉及治炼一轧制一热 
处理等连续的工序, 每个工序需设定关键指标和 工艺参数的控制范围, 才能确保最终产品的质量。 假设, 冶炼工序的关键指标(钢中主要成分)为 $A 1$ 、 $A 2 、 A 3 、 \cdots$, 轧制工序的关键工艺参数(轧制工序 的轧制温度)为 $B 1 、 B 2 、 \cdots$, 热处理工序(热处理 温度)为 $C 1 、 C 2 、 \cdots 、 C m$, 所有工序总计有 $P$ 维 变量。

设各工艺参数的上、下限范围为

$$
\begin{aligned}
& A 1\left[X_{\text {low }}^{1}, X_{\text {up }}^{1}\right], \\
& A 2\left[X_{\text {low }}^{2}, X_{\text {up }}^{2}\right], \\
& \ldots . . . \\
& B 1\left[X_{\text {low }}^{i}, X_{\text {up }}^{i}\right], \\
& B 2\left[X_{\text {low }}^{i+1}, X_{\text {up }}^{i+1}\right], \\
& \ldots \ldots . . \\
& C m\left[X_{\text {low }}^{p}, X_{\mathrm{up}}^{p}\right],
\end{aligned}
$$

则, 所设定的各工艺参数的上、下限是软超球体中 最大的内接矩形体 $F$

$$
\begin{aligned}
F= & (A 1 \cap A 2 \bigcap A 3 \cap \cdots) \\
& \cup(B 1 \cap B 2 \bigcap B 3 \cap \cdots) \\
& \cup(C 1 \cap C 2 \cup C 3 \cap \cdots)
\end{aligned}
$$

A1、A $2 \cdots, B 1, B 2 \cdots, C 1, C 2 \cdots \in F$

$F \in R^{p}$

式中, 第 $i$ 个工艺参数的下限为 $X_{\text {low }}^{i}$, 上限为 $X_{u p}^{i}$ 。

需要说明, “质量可控区” 是指软超球体边界, 而 “工艺规范” 仅指软超球体内最大的内接超矩形 体边界, 如图 3 所示。在软超球体中, 除了内接超 矩形体外, 剩余的区域表示通过工艺参数的优化组 合仍可能满足产品质量要求的可控区域。但是, 当 出现这种情况时, 在设定后续工序的工艺参数时需 进行优化和验证, 确保后工序所设定的工艺参数是 否在软超球体内, 否则容易造成产品质量的异常。

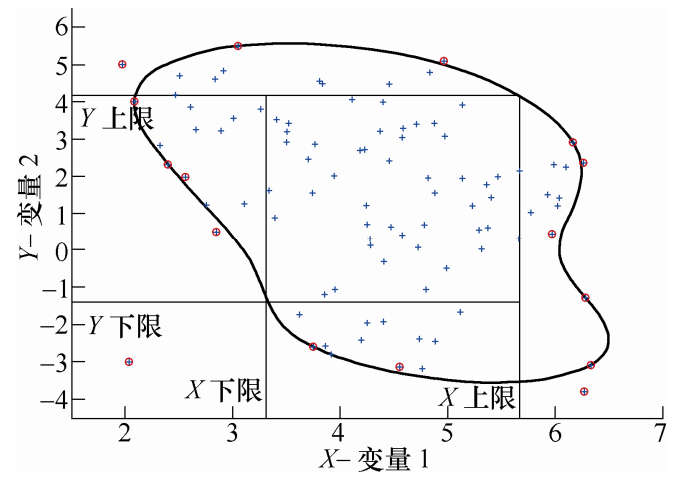

图 $3 X-Y$ 投影面上变量的上、下限

\section{2 工业应用实例分析}

为了验证采用上述所提出的工艺规范制定方法
的有效性, 对实际工业数据进行分析与验证。从实 际生产数据中采集 IF 钢中不同等级汽车钢板在各 生产工序中的主要工艺参数, 包括钢中主要成分、 热轧和热处理过程中关键参数, 如表 1 所示。

表 1 主要工艺参数名称及统计值

\begin{tabular}{lccc}
\hline \multicolumn{1}{c}{ 变量 } & 最大值 & 最小值 & 平均值 \\
\hline $\mathrm{C}(\%)$ & 0.0027 & 0.0011 & 0.0017 \\
$\mathrm{Mn}(\%)$ & 0.160 & 0.100 & 0.128 \\
$\mathrm{P}(\%)$ & 0.014 & 0.007 & 0.010 \\
$\mathrm{~S}(\%)$ & 0.0139 & 0.0024 & 0.0079 \\
热轧加热炉出口温度/ ${ }^{\circ} \mathrm{C}$ & 1277.8 & 1247.5 & 1263.4 \\
精轧入口温度 $/{ }^{\circ} \mathrm{C}$ & 1084.3 & 1021.9 & 1039.8 \\
精轧出口温度 $/{ }^{\circ} \mathrm{C}$ & 927.2 & 898.7 & 916.6 \\
卷取温度 $/{ }^{\circ} \mathrm{C}$ & 753.1 & 655.6 & 708.0 \\
连退加热温度 $/{ }^{\circ} \mathrm{C}$ & 846.9 & 790.9 & 823.5 \\
连退均热温度 $/{ }^{\circ} \mathrm{C}$ & 843.9 & 798.4 & 825.7 \\
连退快冷出口温度 $/{ }^{\circ} \mathrm{C}$ & 455.7 & 351.1 & 432.8 \\
连退时效出口温度 $/{ }^{\circ} \mathrm{C}$ & 394.1 & 346.1 & 375.5 \\
连退缓冷出口温度 $/{ }^{\circ} \mathrm{C}$ & 665.4 & 603.7 & 642.4 \\
\hline
\end{tabular}

\section{1 数据清洗过程}

从工业生产中获取的数据通常存在各种不确 定因素所造成的偏差，在数据分析时需对数据先 进行清洗 ${ }^{[16]}$ 。清洗的目的是剔除数据中的异常点 和强相关的过程变量。数据清洗过程包括对工业 数据进行相关分析和异常点分析。对实际生产线 上采集的 159 个样本点进行基于软超球体的异常 点识别, 发现第 29 个样本点远超出了控制限 $d(x)$, 该控制限可由式(7)计算得到, 结果如图 4 所示。 查看第 29 样本可以发现, 连退快冷出口温度为 $351.1^{\circ} \mathrm{C}$, 远低于平均值 $432.8^{\circ} \mathrm{C}$, 因此将该样本 点从样本集中剔除。

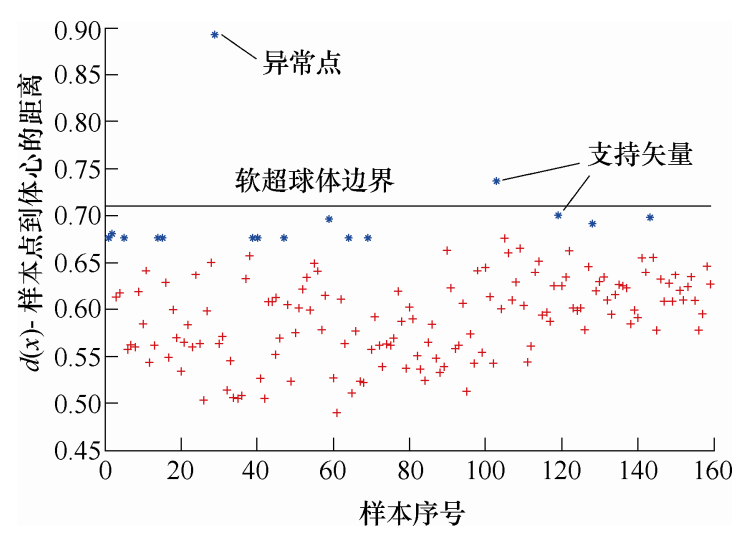

图 4 实际工业数据异常点识别结果

除剔除样本集中的异常点外, 数据清洗过程 还需对各工序中工艺参数间进行相关分析。相关 分析的目的是排除强相关的工艺参数, 因为如果 相邻工序间的工艺参数是强耦合的, 说明这两个 
参数间存在必然性。例如, 热处理工序中连退加 热温度与连退均热温度之间的相关系数为 0.95 , 说明这两个变量是强相关的。原因在于连退炉中 加热温度是依据均热温度的要求来设定的, 它们 之间存在必然性。因此, 为了简化工艺规范制定 过程, 只考虑连退均热温度, 而将连退加热温度 作为关联变量。表 2 给出了热处理工序中其他工 艺参数间的相关系数, 从表中可以看出: 快冷温 度与时效温度、快冷温度与缓冷温度间相关系数 分别为 0.72 和 0.61 , 其他参数对间的相关系数均 小于 0.5 。实际工业应用中, 在制定各工序的工艺 规范时, 主要针对相关系数小于 0.5 的参数对来 确定工艺参数上、下限。涉及到强相关的参数设 定以及相关系数大于 0.5 的工艺参数的设定问题 将在第 3 部分中进行讨论。

\section{表 2 热处理工序各工艺参数间的相关系数}

\begin{tabular}{ccccc}
\hline & 均热温度 & 快冷温度 & 时效温度 & 缓冷温度 \\
\hline 均热温度 & 1.0 & 0.12 & 0.10 & 0.10 \\
快冷温度 & - & 1.0 & 0.72 & 0.61 \\
时效温度 & - & - & 1.0 & 0.43 \\
缓冷温度 & - & - & - & 1.0 \\
\hline
\end{tabular}

\section{2 工艺规范制定过程}

钢材的力学性能指标主要涉及钢中的成分、组 织结构和工艺参数。在 IF 钢生产过程中, 钢的成分 取决于治炼过程中对钢水的合金元素和有害元素的 控制; 轧制工序主要控制轧材的尺寸精度和内部的 组织结构; 热处理工序通过设定不同时间段的温度 来控制材料内部的组织结构。如表 1 所示, 钢的成 分主要包括: C、 Mn、P、S 等; 热轧工序的工艺参 数是热轧加热炉出口温度、精轧入口温度、精轧出 口温度、卷取温度; 热处理工序工艺参数是连退均 热温度、连退快冷出口温度、连退时效出口温度和 连退缓冷出口温度。在这里, 以热处理工序中的工 艺规范制定过程为例, 按照如下步骤来完成工艺规 范的制定。

(1) 利用相关分析方法获得如表 2 所示的变量 间的相关系数。从表 2 中可以看出, 只有 4 对变量 的相关系数小于 0.5 , 由此可以确定该工序的关键变 量, 并两两组合构成待分析的参数对。

(2) 选定高斯核函数, 利用式(7)对数据集进行 学习, 确定工艺参数集的软超球体的边界, 即质量 可控区的边界，并剔除数据集中异常点。

(3) 将软超球体在二维参数对上进行投影, 从 投影的边界中寻找最大内接矩形, 由此来初步确定
工艺参数上、下限。

(4) 根据式(10), 从每个工艺参数初选的上、下 限中确定交集，在此基础上，取参数对之间的并集 来确定各工艺参数的上、下限。

(5) 最后针对该工序装备的过程能力, 对工艺 参数的上、下限作适当调整与优化，最终制定出该 工序的工艺规范标准。

\section{3 工艺规范制定的结果}

按照上述方法, 图 5 给出了热处理工序中, 软 超球体在不同参数对上投影的边界，由此可以确定 均热温度、快冷温度、时效温度、缓冷温度等关键 变量的上、下限。

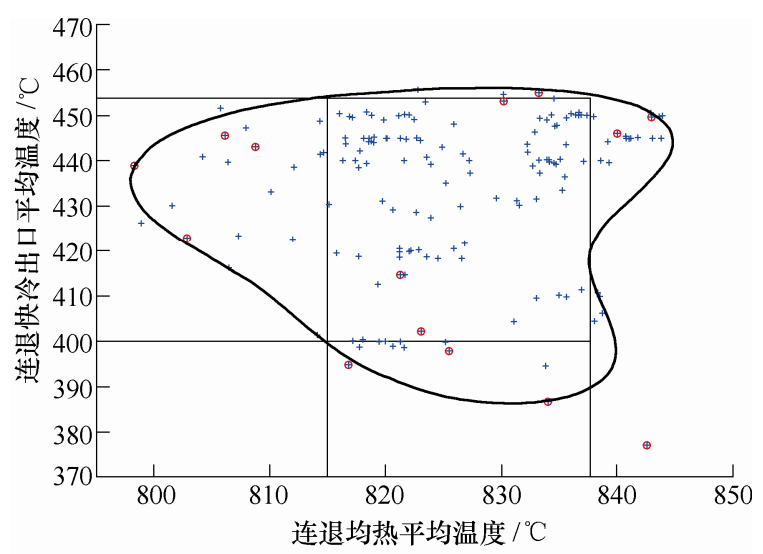

(a) 均热温度 / 快冷温度的上、下限

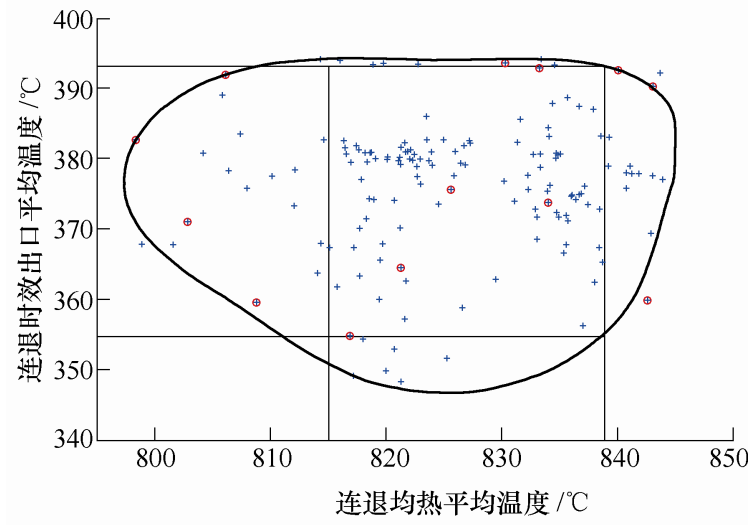

(b) 均热温度 / 时效温度的上、下限

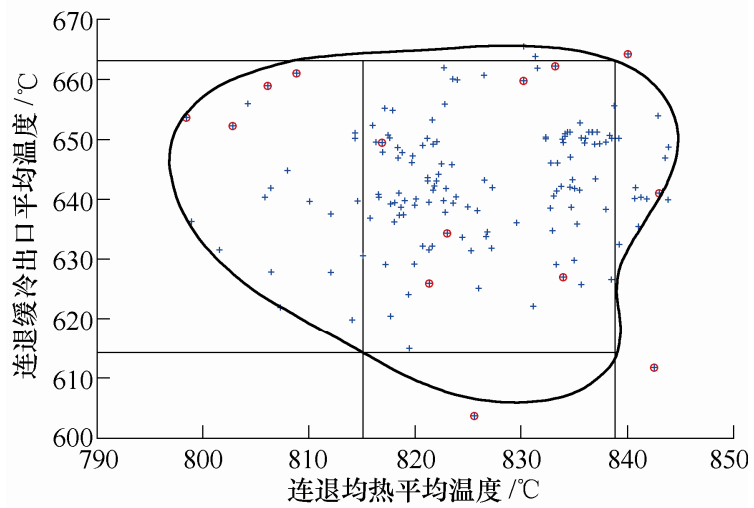

(c) 均热温度 / 缓冷温度的上、下限 


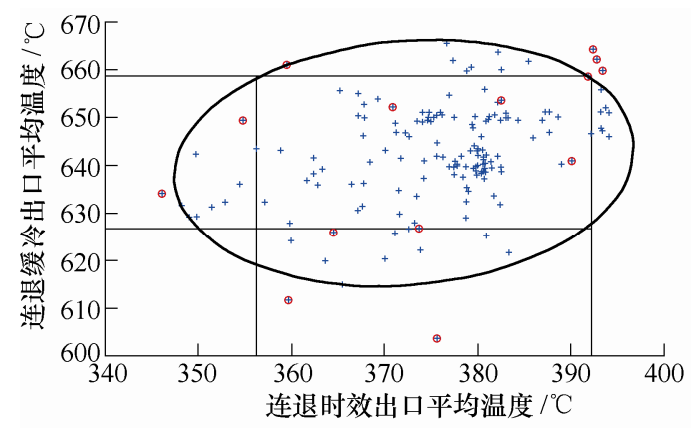

(d) 时效温度 / 缓冷温度的上、下限

图 5 热处理工序主要工艺参数的上、下限

按照对热处理工序的处理方法, 分别确定冶炼 工序及热轧工序的工艺参数的上、下限。表 3 给出 了经优化和验证后, 各工艺参数的上、下限范围, 以及根据 6-Sigma 方法设定的上、下限。

表 3 主要工艺参数的上、下限边界

\begin{tabular}{lcc}
\hline \multicolumn{1}{c}{ 变量 } & 软超球体上、下限 & 6-Sigma 上、下限 \\
\hline $\mathrm{C}(\%)$ & $0.0018 \sim 0.0024$ & $0.0008 \sim 0.0026$ \\
$\mathrm{Mn}(\%)$ & $0.100 \sim 0.160$ & $0.0799 \sim 0.1759$ \\
$\mathrm{P}(\%)$ & $0.0065 \sim 0.0115$ & $0.0048 \sim 0.0156$ \\
$\mathrm{~S}(\%)$ & $0.0048 \sim 0.0118$ & $0.0025 \sim 0.0133$ \\
加热炉出口温度 $/{ }^{\circ} \mathrm{C}$ & $1255 \sim 1273$ & $1246 \sim 1280$ \\
精轧入口温度 $/{ }^{\circ} \mathrm{C}$ & $1030 \sim 1055$ & $1013 \sim 1067$ \\
精轧出口温度 $/{ }^{\circ} \mathrm{C}$ & $908 \sim 924$ & $907 \sim 926$ \\
卷取温度 $/{ }^{\circ} \mathrm{C}$ & $685 \sim 740$ & $582 \sim 834$ \\
连退均热温度 $/{ }^{\circ} \mathrm{C}$ & $815 \sim 838$ & $793 \sim 857$ \\
连退快冷出口温度 $/{ }^{\circ} \mathrm{C}$ & $400 \sim 453$ & $376 \sim 489$ \\
连退时效出口温度 $/{ }^{\circ} \mathrm{C}$ & $356 \sim 393$ & $345 \sim 407$ \\
连退缓冷出口温度 $/{ }^{\circ} \mathrm{C}$ & $613 \sim 663$ & $609 \sim 675$ \\
\hline
\end{tabular}

从表 3 中可以看出, 采用软超球体边界所确定 工艺参数的上、下限区域均比基于 6-Sigma 所确定 的上、下限区域要严格, 尤其是 $P($ 磷) 和 $S$ (硫)这两 个有害元素的上限被严格控制, 说明新方法要比传 统的方法更加合理和精准。

\section{3 讨论}

工艺规范的制定过程应根据产品质量标准和用 户需求, 在实践中不断优化和验证。在实际工业应 用中, 下面几个问题需进一步讨论。

\section{1 关键变量的选择}

流程工业在产品制造过程中涉及多个连续相关 的工序, 每道工序需精准地控制多个工艺参数, 才 能确保产品最终质量。在选择哪些工艺参数作为工 艺规范的关键变量时, 应将那些与产品质量密切相 关的关键指标和工艺参数作为关键变量。在遴选过 程中, 可以通过互相关分析来判定工艺参数与产品 质量的关联度, 并选择相关系数绝对值较大的工艺
参数作为工艺规范需优化的关键变量。

变量选择的另一个问题是强相关的工艺参数的 舍取问题。工艺参数间出现强耦合的主要原因在于 相邻工序之间缺乏可以控制的手段或没有必要加以 控制, 因而上工序的工艺参数被自然地延续到下工 序，如第 2.1 节所讨论的热处理工序中加热温度与 均热温度。将两个强耦合的工艺参数都选为关键变 量, 将会造成软超球体在该变量对上的投影缩成一 条线, 这给工艺规范的制定带来不必要的麻烦。因 此, 在选择变量时, 应剔除其中一个强耦合的变量。 此外, 如果某一重要的工艺参数的方差接近零, 说 明该工艺参数在生产过程没有变化, 可以直接设定 该参数的工艺规范, 而无须通过软超球体来确定工 艺规范。

\section{2 制定相关变量的工艺规范}

实际工业生产中，经常存在变量间多重耦合问 题。如果变量间相关系数较大, 同样会造成软超球 体的边界在该变量对的二维投影变窄。因此, 当变 量间的相关系数大于 0.5 时, 原则上不再以该变量 对的投影边界作为确定变量上、下限的依据, 而作 为验证的手段。为了解释这个问题, 以 2.1 节讨论 的热处理工序中快冷出口温度与时效出口温度为 例。该变量对的相关系数为 0.72 , 因此没有选择该 变量对来确定变量的上、下限。

从图 5 中可以看到, 时效出口温度的上、下限 为 $\left[356{ }^{\circ} \mathrm{C}, 393^{\circ} \mathrm{C}\right]$, 快冷出口温度的上、下限为 $\left[400^{\circ} \mathrm{C}, 453^{\circ} \mathrm{C}\right]$ 。为了进行比较分析, 将软超球体 投影到这两个变量的二维平面上, 且以快冷出口温 度的上、下限 $400^{\circ} \mathrm{C}$ 和 $453^{\circ} \mathrm{C}$ 作为参照点, 分别计算 它们在时效出口温度坐标上对应的边界点为 $348{ }^{\circ} \mathrm{C}$ 和 $393{ }^{\circ} \mathrm{C}$, 如图 6 所示。从图中可以看出, 两个不同的投影面所确定的变量上、下限基本一致, 唯一的差别在于: 图 6 中所确定的时效出口温度的 下限偏低, 增加了工艺规范的区间范围。

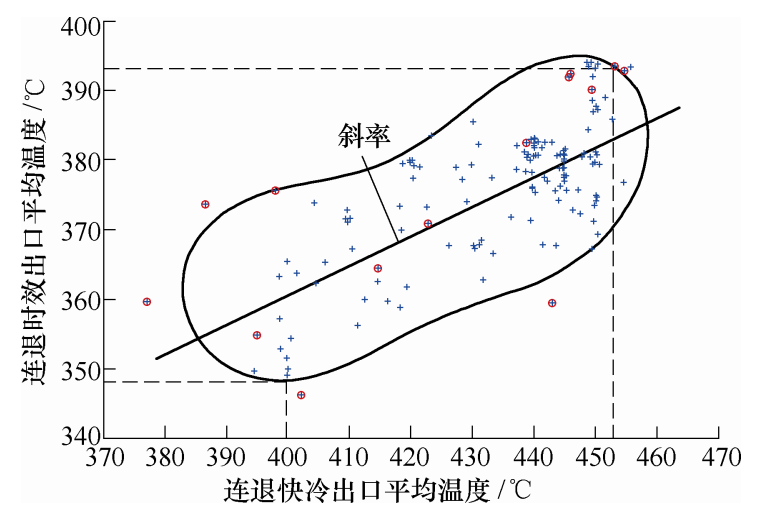

图 6 快冷温度/时效温度的二维投影图

通过这个实例分析可以说明，不同投影面上所 
确定的变量上、下限存在内在的对应关系, 其实质 是软超球体中内接矩形体在不同方向上的投影。因 此, 在确定变量上、下限时, 可以选择相关系数较 小的变量对作为判定的主要对象, 而将相关系数较 大的变量对作为验证和优化的判据。从图 6 中还可 以看出, 当相关系数较大时, 软超球体边界的投影 变得细长, 且倾斜。这两个因素均使得内接矩形变 小, 这会给工艺规范的制定带来影响。实际上, 相 关系数不仅与变量的变化斜率(倾斜程度)有关, 还 与变量的变化方差相关。

\section{3 工艺规范的优化和验证}

虽然从概念上讲, 工艺规范制定过程是从软超 球体中寻求最大的内接矩形体, 但在实际工业应用 中还应考虑到该方法的可操作性。由于变量之间的 多重耦合, 求解最大的内接矩形体的过程不仅仅是 一个复杂的组合优化问题, 而且涉及所制定的工艺 规范是否具有可行性。因此, 在制定工艺规范时应 考虑以下几个重要问题。

(1) 工艺装备条件: 由于工艺装备的过程能力 决定了工艺规范可操作的范围 ${ }^{[9]}$, 因而在制定工艺 规范时首先需满足工艺装备的过程能力这个基本约 束条件。在满足该工艺装备的过程能力的前提下, 优化其工艺参数的控制范围。此外, 在工艺规范制 定过程中, 还应同时考虑其他可优化的条件, 如工 艺流程优化、操作者的操作水平等诸多因素。尤其 对于质量要求更严格的高端产品, 在实施工艺规范 时, 还需额外设定合适的工艺流程, 且确保工艺装 备的服役状态能满足所需的过程能力。

(2) 组合优化问题: 从集合论上来说, 寻求软 超球体中最大的内接矩形体是一个最大集合和最小 集合的组合优化问题。最大集合是指软超球体在二 维投影平面上寻求最大的内接矩形, 而最小集合是 指所有从二维投影所求得的内接矩形中寻求变量的 最小并集。对于具有复杂边界的软超球体, 二维投 影平面上的最大内接矩形集合中的最小集合不一定 是全域最优解。因此, 在实际工业应用中, 重要的 是寻求可行解。有关这类组合优化问题的全域最优 解还有待进一步研究。

(3) 工艺规范的验证: 所提出的工艺规范制定 方法是基于数据驱动的分析技术, 因此数据的可靠 性和完备性对分析结果有着重大影响。数据除了需 清洗外, 还应验证数据的可靠性, 确保数据准确、 可靠。在数据的完备性方面, 不要因为忽略了某些 重要变量而造成产品质量的重大偏差。数据的完备 性还应考虑训练数据是否涵盖了所有区域范围, 尤 其是收集出现质量异常的区域。包含一定数量异常
点的训练数据集更有利于准确地划分软超球体的边 界, 因而有利于正确地制定工艺规范。总之, 制定 工艺规范是一个不断优化、验证的过程。

\section{4 结论}

流程工业在产品制造过程中涉及多个连续相关 的工序, 每道工序应精准的控制相关的工艺参数, 因而需制定产品的工艺规范。但是, 由于工艺参数 间存在多重相关和非正态分布的数据特征，因此基 于参数独立同分布假设的传统 6-Sigma 方法所确定 的工艺规范会造成产品质量的偏差。针对流程工业 目前在工艺规范制定过程中存在的问题, 提出了基 于多元统计过程分析原理的工艺规范确定方法。主 要结论如下:

（1）引入了软超球体的概念，并采用非线性核 函数的方法将原始空间的样本点映射到高维特征空 间中, 通过计算特征空间中的样本点间的内积来确 定软超球体在高维空间上的边界。软超球体方法能 更准确地划定质量可控区的复杂边界, 较好的解决 了工艺参数间存在多重相关和非正态分布情况下多 元变量的统计过程分析问题。

(2) 提出了通过寻求软超球体最大内接矩形体 的方法来制定工艺规范的方法。首先, 将软超球体 投影到各方向的二维平面上, 寻求最大的内接矩形, 然后从所有二维投影所求得的内接矩形中寻求变量 的最小集合, 即软超球体的最大内接矩形体。

(3) 结合 IF 钢生产实例, 讨论了工艺规范制定 的整个过程以及需关注问题。重点讨论了数据清洗 方法、多重相关情况下工艺规范制定以及工艺规范 的优化和验证等相关问题。制定的工艺规范已在实 际生产中被验证。

\section{参 考 文 献}

[1] THOMPSON J R, KORONACKI J. Statistical process control for quality improvement[M]. New York: Chapman and Hall, 1993.

[2] ANDERSON T W. An introduction to multivariate statistical analysis[M]. New York: Wiley, 1984.

[3] JOHNSON R A, WICHERN D W. Applied multivariate statistical analysis[M]. Englewood Cliffs NJ : Prentice-Hall, 1998

[4] FUCHS C, KENETT R S. Multivariate quality control[M]. New York: Dekker, 1998.

[5] KANO M, HASEBE S, HASHIMOTO I, et al. A new multivariate statistical process monitoring method using principal component analysis[J]. Computers \& Chemical 
Engineering, 2001, 25(7-8): 1103-1113.

[6] TAX D M J. One-class classification[D]. Delft: Delft University of Technology, 2001.

[7] SAKLA W, CHAN A, JI J, et al. An SVDD-based algorithm for target detection in hyper spectral imagery[J]. Geoscience and Remote Sensing Letters, IEEE, 2011, 8(2): 384-388.

[8] LIU B, XIAO Y, CAO L, et al. SVDD-based outlier detection on uncertain data[J]. Knowledge and information systems, 2013, 34(3): 597-618.

[9] 徐金梧. 冶金生产过程质量监控理论与方法 $[\mathrm{M}]$. 北 京：冶金工业出版社，2015.

XU Jinwu. Quality monitoring theory and method of metallurgical production process[M]. Beijing : Metallurgical Industry Press, 2015.

[10] SHAWE-TAYLOR J, CRISTIANINI N. Kernel methods for pattern analysis[M]. Beijing: China Machine Press, 2005.

[11] ROSIPAL R, TREJO C J. Kernel partial last squares regression in reproducing Kernel Hillbert space [J]. Journal of Machine Learning Research, 2001, 2: 97-123
[12] CHOU Y M, MASON R L, YOUNG J C. The control chart for individual observations from a multivariate non-normal distribution[J]. Communications in statistics-Theory and methods, 2001, 30(8-9): 1937-1949.

[13] BACH F R , JORDAN M I. Kernel independent component analysis[J]. Journal of machine learning research, 2002, 3(7): 1-48.

[14] LI X, YANG Y, ZHANG W. Statistical process monitoring via generalized non-negative matrix projection[J]. Chemometrics and Intelligent Laboratory Systems, 2013, 121: 15-25.

[15] BOTEV Z I， GROTOWSKI J F，KROESE D P. Kernel density estimation via diffusion[J]. The Annals of Statistics, 2010, 38(5): 2916-2957.

[16] MASON R L, YOUNG J C. Multivariate statistical process control with industrial application[J]. Technometrics, 2004, 46(4): 484-485.

作者简介: 徐钢(通信作者), 男, 1980 年出生, 博士, 助理研究员。主 要研究方向产品质量建模与监控、信号处理与模式识别。

E-mail: watermoon999@126.com 